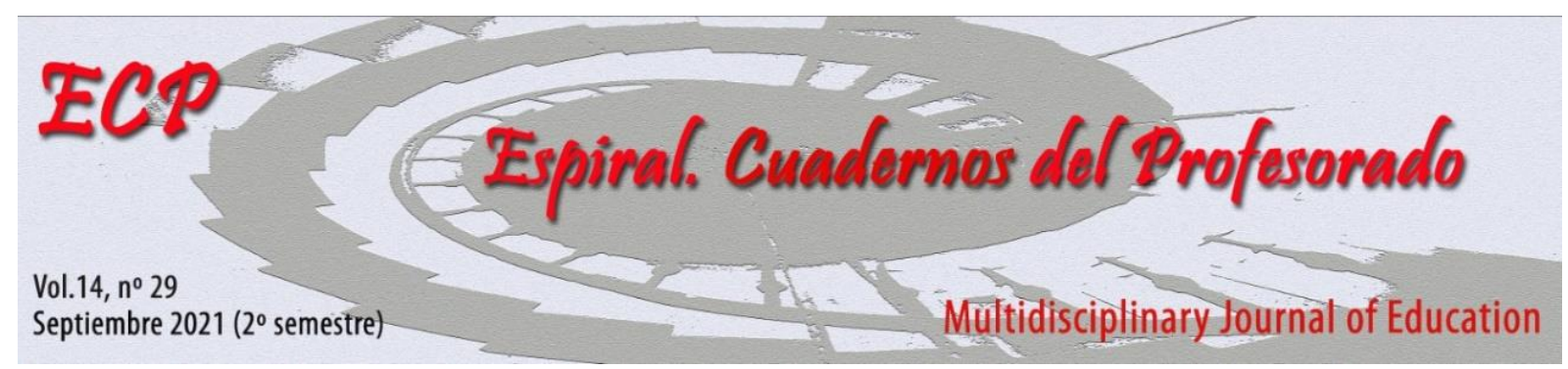

\title{
Adquisición pragmática discursiva en L2/LE: marco conceptual y aplicativo para la educación superior
}

\section{Pragmatic discourse acquisition in L2/FL: conceptual framework and application for higher education}

\author{
Natasha Leal Rivas \\ Università degli Studi di Napoli Federico II, Italia
}

\begin{abstract}
Resumen
El presente estudio propone una reflexión articulada en dos dimensiones, conceptual y aplicativa, que aborda cómo integrar procesos de adquisición pragmática en L2/LE. Para ellos es necesario conceptualizar el modelo de Competencia Comunicativa Intercultural Crítica (CCIC) que cabe entender como un conjunto de conocimientos, competencias y habilidades que transcienden la capacidad de comunicar o establecer relaciones sociales vinculadas al uso lingüístico, ya que desde la comprensión crítica de los textos y una concientización discursiva es capaz de integrar dentro de procesos de enseñanza/aprendizaje lingüístico un "saber comprometerse" socialmente a través del uso crítico de la lengua. El modelo de CCIC pone la atención en el desarrollo consciente de la Competencia Crítica y la Competencia Metapragmática para activar procesos de comprensión crítica y análisis crítico del discurso que facilitan el reconocimiento de esquemas de producción e interpretación ideológica de los mismos, así como de los procesos lingüísticos inferenciales que subyacen en estos. El modelo de CCIC mantiene la centralidad de la competencia discursiva que se identifica con una Competencia Discursiva Crítica (CDC). En el plano aplicativo, y partiendo del estudio génesis que articula una propuesta modelo de CCIC para el aula de ELE (Marimón, 2016), se analiza el alcance de este modelo comunicativo para entornos virtuales de ELE (Leal Rivas, 2018; 2019) a través de propuestas de experimentación acción lingüística que adaptan distintos modelos del Diseño Instruccional con el propósito de sistematizar una perspectiva pragmática en contextos de aprendizaje de ELE para el ámbito académico universitario. El desarrollo del modelo de CCIC en contextos digitales posibilita además la recuperación de corpus textuales (orales y escritos) para su posterior análisis lingüístico que atiende a los fenómenos pragmáticos-discursivos que regulan la creación, funcionalidad e interpretación de los textos y de los géneros discursivos. Los resultados que arrojan los análisis de corpus permiten evaluar el alcance de una eficaz adquisición pragmático-discursiva en L2/ELE en los modelos aplicativos instruccionales para la competencia comunicativa intercultural crítica.
\end{abstract}

Palabras clave: adquisición pragmática; Competencia Comunicativa Intercultural Crítica; análisis discurso; Diseño Instruccional; ELE.

\footnotetext{
Abstract

The present study proposes a reflection articulated in two dimensions, conceptual and applicative, which addresses how to integrate pragmatic acquisition processes in L2/FL. For them it is necessary to conceptualize the model of Critical Intercultural Communicative Competence (CICC) that can be understood as a set of knowledge, skills and abilities that go beyond the ability to communicate or establish social relationships linked to language use, since from the critical understanding of the texts and a discursive awareness is able to integrate within the teaching/language learning processes a "know how to engage socially" through the critical use of the language. The CICC model focuses on the conscious development of Critical Competence and Metapragmatic Competence to activate processes of critical understanding and critical analysis of discourse that facilitate the recognition of
} 
production schemes and ideological interpretation of them, as well as the inferential linguistic processes underlying them. The CCIC model maintains the centrality of discursive competence that is identified with a Critical Discursive Competence (CDC). In the applicative plane, and starting from the genesis study that articulates a model proposal of CICC for the classroom of Spanish Foreign Language (SFL) (Marimón, 2016), the scope of this communicative model for virtual environments of SFL is analyzed (Leal Rivas, 2018; 2019) through experimentation proposals linguistic action that adapt different models of Instructional Design with the purpose of systematizing a pragmatic perspective in SFL learning contexts for the university academic field. The development of the CICC model in digital contexts also allows the recovery of textual corpus (oral and written) for its subsequent linguistic analysis that addresses the pragmatic-discursive phenomena that regulate creation, functionality and interpretation of texts and discursive genres. The results of the corpus analyses allow to evaluate the scope of an effective pragmatic-discursive acquisition in L2/SFL in the instructional application models for critical intercultural communicative competence.

Keywords: pragmatic acquisition; Critical Intercultural Communicative Competence; discourse analysis; Instructional Design, SFL.

Fecha de recepción: 11/02/2021

Fecha de aceptación: 14/09/2021

Natasha Leal Rivas, Università degli Studi di Napoli Federico II, Italia Email: lealrivas@unina.it

\section{Introducción}

El Espacio Europeo de la Educación Superior, con sus logros y deficiencias (Níkleva \& Rodríguez Muñoz, 2015), sigue insistiendo en la necesidad de que los programas formativos universitarios se adecuen metodológicamente a paradigmas competenciales, tanto en la reformulación del rol docente como del estudiante situado en el centro de su propio proceso de aprendizaje como agente activo, responsable y capaz de generar conocimiento interdisciplinar e intercultural con ciertas actitudes hacia el pensamiento crítico (De Miguel, 2009 citado por Baena, 2010).

Desde inicios del S.XXI, el Marco Europeo Común de Referencia para las Lenguas: Aprendizaje, Enseñanza, Evaluación (2002) y el Marco de las Recomendaciones para Competencias Clave en la formación permanente propuesto en 2006 iniciaron a perfilar el contexto de transformación educativa para este siglo XXI. La actualización del Marco de Competencias Claves en 2018 confirmaba la línea de la UE hacia modelos competenciales y enfoques holísticos de enseñanza/aprendizaje. La UNESCO en 2011 ofrecía, además, un instrumento pedagógico para el desarrollo de una Alfabetización Mediática e Informacional (AMI): curriculum para profesores que, desde un enfoque holístico, presentaba un marco de competencias sistematizado y una serie de propuestas de modelos básicos para integrar la AMI en el sistema educativo. La propuesta curricular identifica a los docentes como principales agentes del cambio educativo y de alfabetización, a través de una digitalización responsable. En palabras de Martos Núñez, E. \& Campos F.-Fígares, M. (2013), el desafío competencial docente en un futuro supone saber desarrollar en el aula "un conjunto de competencias clave para evaluar, procesar, usar, comunicar la información a través de medios analógicos y digitales" y ello implica, por supuesto, promover y desarrollar valores sociales, educativos, éticos vehiculados a través del uso digital. La actualización realizada en 2017 sobre el Marco Europeo de Competencias Digitales para la Ciudadanía-DigComp 2.1 y el cuadro de referencia para el desarrollo de una competencia digital docente, el DigCompEdu (2019) cerraban el cuadro completo de recomendaciones y consejos para la promover un educación en competencias en Europa.

Actualmente, el Plan de Acción para la Educación Digital (2021-2027) surgido a raíz de la crisis pandémica de Covid-19 ha puesto de manifiesto el proceso de reformulación de todos los marcos hasta aquí esbozados, y sigue reforzando el enfoque competencial concediendo una importancia destacada a la competencia digital. La Comisión Europea propone para una educación sostenible y de calidad en la era digital dos grandes estrategias. Por un lado, promover un ecosistema educativo digital con altas prestaciones y, por el otro, mejorar las competencias digitales necesarias para una renovación educativa y formación eficaz. Sin embargo, ya antes de la crisis generada por la pandemia de Covid-19,

Espiral. Cuadernos del Profesorado | ISSN 1988-7701 | 2021, 14(29), 73-85 
algunos modelos institucionales para el desarrollo de competencias habían demostrado sus deficiencias de planteamiento y actuación en los distintos niveles educativos. Deficiencias, por ejemplo, que presentan algunos modelos institucionales de competencia digital docente estudiadas en Casañeda, Esteve \& Adell (2018), quienes observan una conceptualización de la noción competencia errónea. Destacan que la "visión taxonómica del concepto de competencia" impide dar cuenta de "la complejidad de los diferentes contextos en los que dicha competencia se pone en acción y se desarrolla y en su papel en la construcción de la identidad del docente". Por otro lado, el contexto pandémico ha agudizado una falta de consenso social sobre las posibilidades de las tecnologías digitales al servicio de la educación.

En todo caso, mientras que los modelos competenciales han tenido un mayor o menor éxito de implementación como modelos institucionales, tanto en proyectos educativos como en programas y diseños curriculares en ámbitos en la educación obligatoria, resulta indicativo la escasa integración teórico-práctica del enfoque por competencias en el ámbito universitario. Sin ser exhaustivos, la autonomía del diseño de los programas docentes, la escasa flexibilidad de los currículums universitarios formativos y los modelos de evaluación estandarizada, entre otras posibles causas no menos representativas, revelan la complejidad del entorno para la implementación del enfoque competencial en numerosas titulaciones universitarias. Ello dificulta una posible transformación de la docencia universitaria que permita evolucionar de la gestión de la información a enseñar un uso responsable del conocimiento.

Enseñar y evaluar por competencias en la universidad es cada vez más necesario si el objetivo de las universidades es ofrecer una formación superior de calidad. Para ello, el balance de la experimentación de modelos competenciales en otros ámbitos educativos puede ofrecer pautas y acciones adecuadamente adaptadas para una implementación con éxito en el ámbito universitario. El perfil competencial académico debe integrar y combinar capacidades, saberes, habilidades, actitudes, experiencia en la toma de decisiones pertinentes, aptitudes relacionales, etc. para saber actuar en contextos particulares de la vida académica y lograr de forma intencional finalidades relevantes. Dicho perfil debe evolucionar hacia un perfil profesional. Ello significa que tales competencias académicas no pueden desempeñarse sólo a través del estudio, sino que necesitan desarrollarse en otros contextos. Para enseñarlas es importante jerarquizar las capacidades, habilidades y destrezas en procesos graduales de integración que llevan al estudiante a un actuar de forma consciente y competente. La evaluación, por tanto, se desarrollaría en términos de actuación.

Con estas breves premisas se ha intentado presentar el estado de la cuestión del marco de contextualización y de actuación donde se justifican los siguientes interrogantes de investigación lingüística aplicada a la enseñanza/aprendizaje de L2/LE en el ámbito de la universidad: ¿Cómo mejorar el perfil competencial en la Educación Superior desde el aprendizaje lingüístico en L2/LE y que ello responda, además, a las necesidades específicas académicas y discursivas de los aprendientes? ¿De qué manera sistematizar una perspectiva pragmática en contextos de aprendizaje de L2/LE para mejorar un aprendizaje funcional y discursivo de la lengua real y competente? ¿Qué modelo de Competencia Comunicativa puede contribuir hacia una competencia discursiva crítica integrando otras competencias académicas que mejoren el futuro perfil profesional? ¿Un modelo de competencia comunicativa intercultural cómo puede desarrollar la construcción y transmisión de un pensamiento crítico y vehicular este a través de un uso social responsable de la lengua?

El presente estudio nace de precedentes investigaciones y experimentaciones lingüísticas para el ámbito universitario con el propósito general de mejorar la calidad de los procesos de aprendizaje lingüístico para Español Lengua Extranjera (ELE). Calidad de las investigaciones que residen en la "interacción continua entre teoría y práctica a través de la observación, investigación, innovación, reflexión y evaluación sobre la misma, llevada a cabo en el contexto institucional, donde los docentes desempeñan su labor" (Imbernón, 2013, citado por López Gómez y Pérez Navío, 2013: 39). En este sentido, el constructo teórico sólido del estudio se asiente sobre el debate interdisciplinar que generan los enfoques funcionalistas, pragmáticos y discursivos de la Lingüística Aplicada y de la Pragmática en ELE (Escandell, 1996; Calsamiglia/Tusón, 1999; Pons, 2005; Ruiz Gurillo, 2006), la Pragmalingüística (Arzamendi, 2005; Fontich, 2006), la Lingüística Cognitiva (Cuenca \& Hilferty, 1999; Castañeda y Alonso, 2009), la Lingüística Sistémico Funcional (Halliday, 1982; Halliday \& Hasan, 1989; Halliday

Espiral. Cuadernos del Profesorado | ISSN 1988-7701 | 2021, 14(29), 73-85 
\& Matthiesen, 2004) y el Análisis del Discurso (Van Dijk, 1999; 2006).

Los estudios citados postulan el fundamento de la relación dialéctica entre forma y función de la lengua y en términos de Halliday (1982: 45, 51 y 66) cómo la creatividad en el lenguaje consiste en la habilidad de un actor social para crear, en nuevos contextos de situación, otros significados a partir de las opciones semánticas que han sido codificadas como opciones gramaticales. Conocer y comprender las normas y convenciones implícitas que subyacen en cualquier interacción social, verbal o no, predispone al hablante al éxito comunicativo. Estas junto a los rasgos cognitivos que los identifica son variables que inciden en los procesos de selección lingüística y discursiva. Rasgos o marcas que se manifiestan ampliamente en los espacios de intersubjetividad lingüística y que, por tanto, lejos de ser meras construcciones cognitivas abstractas, estas se concretan y responden a un esquema más o menos sistematizado que condiciona la eficacia de las habilidades lingüísticas.

Por tanto, este estudio propone una aproximación conceptual y aplicativa que aborda cómo integrar de manera eficaz procesos de adquisición pragmática en L2/LE. Ello es posible desde la experimentación acción lingüística. Se presenta un esquema de conceptualización descriptiva del modelo de Competencia Comunicativa Intercultural Crítica (CCIC) en cuanto este desarrolla un conjunto de conocimientos, competencias y habilidades que transcienden la capacidad de comunicar o establecer relaciones sociales vinculadas al uso lingüístico con el propósito que el hablante sea capaz también de construirse socialmente hacia valores y actitudes de ciudadanía desde la propia competencia discursiva. Para ello se toma como estudio de referencia un estudio y propuesta para el aula de ELE (Marimón, 2016) que articula desde el análisis de discurso crítico y de género, un desarrollo competencial discursivo que integra en el aula un "saber comprometerse" del aprendiente mediante un uso de la lengua socialmente implicado. El modelo de CCIC pone la atención en el desarrollo consciente de la Competencia Crítica y la Competencia Metapragmática para una Competencia Discursiva Crítica (CDC), desde procesos de comprensión crítica y análisis crítico de los textos que facilitan el reconocimiento de esquemas de producción e interpretación ideológica de los mismos. El modelo acoge los postulados de variabilidad lingüística que se rigen, según los postulados funcionalistas de Halliday (1982: 62-63), por tres macro-funciones: la ideativa, la interpersonal y la textual. Estas se articulan en el modelo de CCIC de forma consciente a través de la Competencia Crítica y Competencia Metapragmática. Por un lado, la función ideativa se relaciona con el significado experiencial de los textos en cuanto el lenguaje es un medio de expresión de nuestra experiencia (exterior o interior). La segunda función, la interpersonal, atiende a la intención comunicativa y al plano enunciativo de los textos, revelando los procesos lingüísticos de inferencia, codificación e interpretación, textual y los esquemas de interacción y relación social. Y, por último, la función textual que atiende a la macro y micro estructura discursiva de los textos, es decir, el hablante estructura lo que dice, de manera que tenga sentido dentro de un contexto concreto y en relación a la tipología textual y género discursivo.

La dimensión aplicativa del estudio se centra en el análisis de varios modelos de Diseño Instruccional, modelos desarrollados para procesos de aprendizaje lingüístico en línea que se dividen en fases. Estas facilitan en entornos digitales el desarrollo del modelo de CCIC para una adquisición pragmática más eficaz en contextos de L2/LE. Como estudios de referencia se siguen investigaciones precedentes desarrolladas en contextos virtuales para ELE (Leal Rivas, 2018; 2019) cuyas propuestas de experimentación acción lingüística ofrecen la posibilidad de aunar corpus textuales y analizar posteriormente el alcance de los procesos de adquisición pragmático-discursiva desde la producción textual de los aprendientes.

Otros objetivos del presente estudio se concretan en los siguientes:

1. Definir las competencias que en el modelo de CCIC fomentan la adquisición pragmática en L2/LE, en lo específico la Competencia Crítica y la Competencia Metapragmática. Desde la experimentación activa concreta desarrollada para ELE ha sido posible analizar distintas propuestas aplicativas para el modelo de CCIC que confirman cómo una atenta estructuración de las estrategias (meta)cognitivas favorecen destrezas hacia la adquisición (meta)pragmática en español. Resultan de especial interés estudios específicos de ELE sobre la importancia de la competencia lingüística del docente en la L1 del aprendiente de ELE (Pastor Cesteros, 2004) y la reflexión de los procesos de 
adquisición pragmática desde la transferencia como "fuente de estrategia de aprendizaje" (Galindo Merino, 2016). Alcance: reflexionar cómo integrar una CCrítica articulada en un modelo comunicativo competencial y sistematizada desde procesos de transferencia cognitiva y discursiva en aprendientes no nativos de ELE.

2. Reflexionar desde qué géneros y tipologías discursivas vehicular la adquisición pragmática en entornos virtuales de L2/LE y cuáles son las posibilidades hipermediales e hipertextuales para integrar en contextos digitales un aprendizaje discursivo eficaz para ámbitos especializados como el académico. En esta línea, resultan de gran aportación los estudios interdisciplinares y los paradigmas relacionados con las Nuevas Literacidades y la Digital Literacy que insisten en la necesidad de integrar nuevos códigos de multialfabetización y la convergencia de lenguajes (Martos y Campos, 2012: 6) como nuevos usos sociales digitales de la lengua. El discurso, por tanto, como categoría y eje central en la enseñanza/aprendizaje de la lengua (Campos Fernández-Fígares \& Quiles Cabrera, 2019) hacia una Competencia Discursiva Crítica Alcance: Mejorar la reflexión para integrar procesos de adquisición pragmático-discursiva desde un acercamiento al enfoque de géneros discursivos y tipologías textuales que nos permite comprender mejor las distintas funciones de los actos de habla (verbales y no verbales) en cualquier contexto de uso académico de la lengua.

3. Profundizar sobre los varios modelos de Diseño Instruccional elaborados para procesos de aprendizaje digital desde los postulados de las distintas teorías de aprendizaje cognitivista, constructivista con el propósito de mejorar la calidad de los procesos de aprendizaje lingüístico. Alcance: mejorar los recursos y herramientas que permitan una sistematización pragmática en contextos digitales de enseñanza/aprendizaje de ELE, además de mejorar las habilidades lingüísticas y saber reconocer y desarrollar un uso académico discursivo, atendiendo a necesidades académicas y profesionales futuras.

4. Analizar y comparar el modelo instruccional definido Aprendizaje Colaborativo en Ambientes Distribuidos (ACAD) y sus posibilidades aplicativas. El modelo instruccional ACAD formula un perfil de aprendiente responsable y autónomo con capacidades para transmitir conocimientos, competencias y actitudes desde un cambio de modelos mentales y la adquisición de habilidades. Además, formula un esquema para integrar actitudes y valores de manera organizada. El perfil de aprendiente ACAD comparte con el perfil de aprendiente lingüístico del Plan Curricular del Instituto Cervantes (PCIC) la figura de un agente social, intercultural y autónomo que interrelaciona los distintos "saberes" de forma consciente: aprender a conocer, aprender a hacer, aprender a ser y aprender a convivir. El modelo ACAD además favorece la interdependencia y respeto a las contribuciones dentro de un grupo de aprendizaje, haciendo uso de medios convencionales, de medios sincrónicos o asincrónicos o de plataformas educativas. Alcance: Profundizar en los modelos instruccionales que mejor adapten la sistematización pragmática y discursiva en contextos digitales de ELE y que permitan desarrollar actitudes y valores para que un aprendiente de la lengua aprenda a saber comprometerse y fomente procesos de su construcción social desde su propio aprendizaje y con el uso de la lengua para desarrollar el modelo de CCIC.

Los resultados de investigaciones recientes a partir de la posibilidad de analizar los corpus textuales de los aprendientes de ELE en contextos de aprendizaje lingüístico en línea (Leal Rivas, 2020b) confirman el alcance positivo de la implementación modular del DI como modelo aplicativo para el desarrollo de la CCIC, y la necesidad de seguir perfilando un modelo competencial adecuado para una sistematización pragmática adecuada en ELE y para procesos de aprendizaje lingüístico en línea.

\section{Integrar la adquisición pragmática en L2/LE: Dimensión conceptual del modelo de Competencia Comunicativa Intercultural Crítica}

Los procesos adquisicionales en L1 atienden a la interiorización de los usos sociales de la lengua y a la sistematización de estos en los varios registros y modalidades discursivas de la lengua. En contextos de aprendizaje de L2/LE, es importante volver a contextualizar los procesos de aprendizaje lingüístico dentro de una dimensión adquisicional, no acumulativa como con frecuencia se entiende para la L1, sino desde una dimensión adquisicional identificada con la noción de proceso completo de sistematización de usos sociales de la lengua. Para Rutherford (1987) la adquisición linguiística en L1 comprendía los distintos 
procesos que se desarrollaban desde la asimilación de los usos sociales de la lengua en contextos de socialización, pero también los procesos de adquisición discursiva de estos dentro de un contexto de escolarización. La importancia de transferir procesos de adquisición pragmática-discursiva en L2/LE desde la L1, como en breve se desarrollará, es posible desde un enfoque discursivo y funcional de la lengua.

Los principales modelos de competencia comunicativa han ido posicionando la competencia pragmática del hablante en un lugar relevante, aunque no la citen explícitamente. En estos modelos la competencia pragmática integra tanto las habilidades pragmalingüísticas (saber gestionar los turnos de palabra, ser capaces de desambiguar enunciados, saber elegir actos verbales y no verbales según el nivel de formalidad, la distancia social y las estrategias de cortesía verbal y no verbal, etc.) como las habilidades sociopragmáticas, relacionadas con la actualización de actos de habla según aspectos socioculturales que condicionan la pertinencia y la eficacia pragmática. En concreto, el modelo de competencia comunicativa de enfoque discursivo (Celce-Murcia et al., 1995) y el modelo de competencia comunicativa intercultural (Byram, 2009) permiten el desarrollo de un modelo de Competencia Comunicativa Intercultural Crítica que posibilite integrar procesos sistematizados de adquisición pragmática en L2/LE para una Competencia Discursiva Crítica.

Celce-Murcia et al. (1995) define la competencia accional en cuanto competencia pragmática como la "habilidad para transmitir y entender el intento comunicativo al realizar e interpretar actos de habla y funciones lingüísticas" (1995, p. 17). Es obvio que la competencia pragmática integre una Competencia Metapragmática para desarrollar habilidades comunicativas que se identifican con la capacidad de comprender el proceso intencional comunicativo de un mensaje y su consecuente selección estratégica discursiva y lingüística.

Dentro del modelo del modelo de Competencia Comunicativa Intercultural Crítica (CCIC) la Competencia Metapragmática se activa desde a través de destrezas que permiten conocer cómo funcionan los procesos lingüísticos de inferencia en una lengua a través del esquema de estrategias de producción y recepción, pero estas también se producen desde los esquemas de interacción de los géneros discursivos. De hecho, Portolés (2004) la menciona como la capacidad que tienen los hablantes para seleccionar uno u otro enunciado en función de la intención comunicativa y del contexto situacional comunicativo. También Rodríguez Muñoz (2010) coincide en que "la conciencia metapragmática se concretaría en la capacidad de selección de una formulación apropiada para cada momento de comunicación mediante una serie de destrezas diversas que se recogen en la competencia comunicativa."

En contextos de aprendizaje lingüístico de L2/LE, una mejor formulación sistematizada de la Competencia Metapragmática permitiría integrar adecuadamente procesos de adquisición pragmática vinculados a la construcción discursiva. Ello favorece además una dimensión de comunicación intercultural crítica. La dimensión sistémica funcionalista de Halliday (1982) sostiene que conocer el uso de la lengua y las normas socioculturales que la rigen desde una dimensión analítica de los propósitos concretos y específicos de los actos de habla fomenta una perspectiva de análisis interlingǘstica o intercultural. Así, el desarrollo de habilidades metapragmáticas para una CCIC en L2/LE ha de activarse previamente desde estrategias (meta)cognitivas y procesos hacia un uso crítico (no sólo reflexivo) de las funciones pragmáticas y sociales de la lengua conformando una dimensión contrastiva intercultural.

La Competencia Crítica (CCrítica) en el modelo de CCIC se identifica dentro de la competencia accional y la competencia estratégica definida por Celce-Murcia y será la promotora para activar procesos adquisicionales pragmático-discursivos. Halliday \& Hasan (1989) definen esta habilidad crítica como un "aprender a significar", es decir, como la capacidad de aprender a observar, analizar y reconocer los esquemas que desarrolla una lengua en función del uso social. Por tanto, observar, analizar y reconocer serán procesos cognitivos que habrá que fomentar en el modelo de CCIC de forma consciente para "aprender a significar" en contextos de aprendizaje lingüístico. La CCrítica permite desarrollar procesos de concienciación crítica de la (meta)lengua, que van más allá del valor de reflexionar el uso de la lengua. Por lo tanto, el hablante es consciente de poseer habilidades para un uso crítico de la lengua y un saber aprender la lengua que, sin duda, se espera pueda incidir en un saber ser y saber comprometerse socialmente utilizando la lengua. Esta competencia no representa una mera competencia estratégica ya que dentro del modelo de CCIC articula la posibilidad de crear y construir pensamiento crítico. 
¿Qué ámbito aplicativo tiene la CCrítica y de qué manera puede concretarse para integrar procesos de adquisición pragmática en contextos de aprendizaje lingüístico? En primer lugar, tanto el Marco Común Europeo de Referencia para las Lenguas (MCER, 2002) como el Plan Curricular del Instituto Cervantes (PCIC) especifican la relevancia de competencias generales que exceden de las competencias comunicativas. Desde una formulación funcionalista ambos cuadros reconocen un esquema de saberes interrelacionados a las competencias: el saber declarativo (los conocimientos), el saber hacer (las destrezas y habilidades), el saber ser (la competencia existencial) y el saber aprender (la capacidad de aprender). El PCIC específicamente propone un Inventario por niveles de Tácticas y Estrategias pragmáticas. Y si bien el enfoque pragmático de la lengua debe atender a los distintos niveles funcionales, organizativos y discursivos de la lengua, el uso crítico y reflexivo de las normas de uso de la lengua (la CCrítica) representa un componente importante.

Como se ha evidenciado algunas líneas más arriba, la CCrítica se encuentra también dentro de una competencia estratégica ya que la habilidad de saber seleccionar y formular los enunciados ha de realizarse de forma crítica y consciente, atendiendo a los componentes del contexto situacional y de la interacción lingüística. En este sentido, la CCrítica y su valor estratégico del saber aprender posibilita "la capacidad de observar y de participar en nuevas experiencias y de incorporar conocimientos nuevos a los ya existentes, modificando estos cuando sea necesario" (MCER, 2002, p.104).

Al hilo de lo expuesto, el enfoque holístico se presenta como el más adecuado para integrar la adquisición pragmático-discursiva en contextos virtuales de aprendizaje lingüístico (2020a) pues asume "incorporar una perspectiva sociocultural, a la lingüística y a la cognitiva. Esta perspectiva debe adoptar un punto de vista más global, interesándose por los interlocutores, sus culturas, sus organizaciones sociales, las instituciones con las que se vinculan, etc." (Cassany, 2017, p. 7). Ello comporta el desarrollo de actitudes implicadas en un proceso de aprendizaje de la lengua consciente y crítico.

De hecho, la CCrítica en el modelo discursivo de CCIC se articula abarcando cada una de las dimensiones del lenguaje en cuanto proceso cognitivo, lingüístico y social. Un posible modelo aplicativo para L2/LE debe integrar de forma holística cada una de estas dimensiones.

En la dimensión cognitiva, la CCrítica es capaz de activar procesos adquisicionales (no acumulativos) para interiorizar y sistematizar las normas que regulan los usos sociales de la lengua. Se activa desarrollando estrategias (meta)cognitivas para una mejor concienciación de la lengua meta y "debe ser cíclica, holística y orientada al estudiante mediante recursos y estrategias que darán énfasis a la comprensión, a los principios generales para el aprendizaje de la lengua y a la experiencia operativa" (Leal Rivas, 2020a, p. 76). En esta dimensión se articulará además la Competencia Metapragmática a través de la reflexión y uso crítico de la lengua en contextos de interacción comunicativa real.

En la dimensión lingüística, es fundamental para integrar la adquisición pragmática en L2/LE desarrollar procesos de reflexión crítica acerca del funcionamiento de la lengua desde la comprensión crítica. Se atiende al significado experiencial e interpersonal de los textos y/o discursos para una mejor comprensión inferencial e ideológica de estos. La CCrítica en la dimensión lingüística activa procesos de transferencia significativa discursiva: desde los conocimientos previos (Bisquerra, 2015) y la propia sistematización discursiva en L1 la CCrítica construye procesos contrastivos hacia una Competencia Discursiva Crítica (CDC). Ello es posible a partir de un Análisis previo de los aprendientes que permite conocer el dominio discursivo y las necesidades comunicativas y discursivas reales. Desarrollar una CCrítica en la dimensión lingüística desde la comprensión crítica de los textos y el análisis del significado interpersonal y experiencial posibilita una Competencia Discursiva Crítica (CDC) en la que el aprendiente de ELE a través de textos reales aprende a reconocer esquemas pragmáticos de producción e interpretación inferencial y a diferenciar los varios patrones de actualización de los actos de habla según la tipología o género discursivo.

En la dimensión social del lenguaje, la CCrítica se activa en la competencia sociocultural vinculada a la producción de textos (Quiles Cabrera \& Caire, 2013) y al desarrollo de una Competencia Discursiva Crítica (Leal Rivas, 2020 $)$. Atiende a la dimensión intercultural donde los aspectos socio pragmáticos de la lengua cobran mayor relevancia en la construcción discursiva. El aprendiente de L2/LE en contextos de aprendizaje basado en competencias y desde un enfoque holístico, funcional y discursivo, 
desarrolla aspectos de interculturalidad que le hacen "saber ser" y "saber comprometerse" a partir de un uso discursivo de la lengua implicado socialmente. La importancia de los textos y

\section{Integrar la adquisición pragmática en ELE: dimensión aplicativa en entornos virtuales con modelos instruccionales}

La dimensión aplicativa del modelo de Competencia Comunicativa Intercultural Crítica (CCIC) ha de priorizar, por lo tanto, la dimensión discursiva y sistémico-funcional del aprendizaje lingüístico con el fin de integrar de forma eficaz una perspectiva pragmática en L2/LE. Los contenidos lingüísticos, nociofuncionales, comunicativos o culturales pueden articularse desde un análisis funcional de los textos en base a las tres macro funciones: la función ideacional o experiencial, identificada con el contexto comunicativo del texto; la función interpersonal, identificada con la intención comunicativa y los actos de lengua actualizados en el texto; y la función textual que corresponde a la organización del texto (Leal Rivas, 2020a). A priori, es fundamental articular la dimensión cognitiva del aprendizaje lingüístico desde la personalización de estrategias cognitivas que permitan una eficaz comprensión pragmática-discursiva de los textos (Leal Rivas, 2018) que revierte de manera positiva en una trasferencia discursiva para la creación de textos académicos (Leal Rivas, 2020b). Las muestras de textos reales permiten accionar la esfera de la CCrítica. Los textos se prefieren sin adaptar, pero sí seleccionados con atención por el docente en base al dominio lingüístico y a las necesidades comunicativas-discursivas de los aprendientes (Robles Ávila, 2019), facilitando la observación, el reconocimiento y el análisis de los fenómenos pragmáticosdiscursivos (adecuación, cohesión, coherencia, cortesía, cooperación, actitud, etc.) de los textos, así como los mecanismos que rigen la organización textual (Van Dijk, 1999) para un mejor interiorización y uso discursivo del aprendiente.

Se analizan ahora los diversos modelos aplicativos experimentados para integrar procesos de adquisición pragmática aplicado a ELE. La estructuración pedagógica de los varios modelos instruccionales (Díaz Barriga, 2006) y su adaptación para la experimentación en entornos virtuales para ELE (Leal Rivas, 2018; 2019; 2020a, 2020b) han seguido una planificación que responde al enfoque competencial propuesto por el modelo de CCIC como marco conceptual lingüístico.

Cabe precisar antes que la experimentación metodológica de los modelos instruccionales para un modelo aplicativo de la CCIC en entornos virtuales se sustenta en las indicaciones de la UNESCO respecto a la importancia de asegurar la presencia del docente que "debe ofrecer situaciones de aprendizaje, no lecciones, para que el estudiante las recorra extrayendo de su contexto lo que ayude, más allá de ese momento de contacto sincrónico. Con esta continuidad de situaciones de aprendizaje que el estudiante experimenta, reflexiona y evalúa, irá más allá, necesitará reaccionar, moldearse, cambiar. Formará una capacidad de evaluar, seleccionar y aplicar los elementos de su contexto para su aprendizaje o sus restantes actividades. Este será el verdadero aprendizaje en los entornos virtuales." (Álvarez Ramos, 2017). Es importante precisar que los modelos y procesos de aprendizaje en entornos virtuales creados por el docente deben igualmente respetar las dimensiones de "desarrollo emocional, moral, social, espiritual, estético y motivacional, en tanto que los componentes son: conocimiento, capacidades y actitudes, los cuales apuntan tanto al desarrollo intrapersonal como interpersonal del individuo". Compartimos con Iván De león C. y José Suárez (2008) que el modelo de diseño Instruccional aplicado al aprendizaje de lenguas, debe garantizar la interacción docente-alumno, alumno-alumno

Para formar aprendientes lingüísticos capaces de desenvolverse discursivamente de forma eficaz en el propio ámbito académico en lengua extranjera, se piense en los estudiantes Erasmus que deben desarrollar parte de su ciclo académico en contextos académicos a veces no homogéneos, es necesario elaborar itinerarios de aprendizaje lingüístico que tengan en cuenta la formación de "lectores expertos" (Martínez Ezquerro y Martos García, 2019: 21). El diseño y la estructura pedagógica de un modelo que integre procesos de adquisición pragmática debería articular prácticas lectoras interculturales que posibilitan el desarrollo de habilidades lingüísticas capaces de desenvolverse ante las nuevas literacidades del siglo XXI (Martínez Ezquerro, 2020). Ello supone, sin duda, un esfuerzo considerable para el docente que debe continuamente adaptar los distintos itinerarios de aprendizaje lingüístico según necesidades y competencias pre-adquiridas. En cierto modo, con una adecuada competencia digital docente los entornos virtuales pueden facilitar algunas tareas de re-adaptación y reutilización de los itinerarios. Ello como se ha

Espiral. Cuadernos del Profesorado | ISSN 1988-7701 | 2021, 14(29), 73-85 
destacado hasta aquí es posible si el diseño pedagógico sigue una sólida fundamentación teórica lingüística desde un modelo competencial adecuado para integrar de forma realística un enfoque holístico y competencial, que no siempre es fácil de diseñar y que, además, para los entornos virtuales requiere también una determinada competencia digital del aprendiente.

A continuación, se describen brevemente los modelos instruccionales experimentados para el desarrollo del modelo de CCIC y la dimensión pragmático-discursivo en ELE en entornos digitales.

El modelo ADDIE (Leal Rivas, 2018; 2019) es el modelo básico de Diseño Instruccional interactivo, en donde los resultados de la evaluación formativa de cada fase pueden llevar al docente a reformular cualquiera de las fases previas. Sus fases se identifican con el Análisis, Diseño, Desarrollo, Implementación y Evaluación. La propuesta didáctica se centra en el desarrollo de competencias discursivas para un grupo específico de aprendientes de ELE, del ámbito de la Medicina y de las Ciencias de la Salud, cuyo objetivo era mejorar la competencia discursiva oral en la interacción comunicativa entre médico/paciente y la interacción profesional entre médico/médico.

Otro modelo instruccional, derivado del primero, es el modelo de Investigación Acción Participativa (IAP) de Wadsworth (1998, citado por Martí, 2002), que, a su vez, supone una evolución del modelo de Investigación Acción de Kemmis y McTaggart (1988, citado por Elliott, 1993). El modelo IAP se adapta para desarrollar la CCIC y la adquisición pragmática-discursiva en aprendientes no nativos de español con dominio básico de la lengua, pero con necesidades académicas discursivas específicas. Los aprendientes/participantes a la IAP, estudiantes Erasmus que necesitaban mejorar su perfil competencial académico en español y de escritura académica, conformaron un corpus textual en el que fue analizado los sobre los (sub)procesos cognitivos del perfil competencial de escritura académica (Leal Rivas, 2020b). El estudio confirma el alcance positivo del modelo IAP para el desarrollo de la CCIC y para mejorar la competencia discursiva escrita con una adecuada sistematización pragmática en contextos de aprendizaje lingüístico en línea.

El modelo de Aprendizaje Colaborativo en Ambientes Distribuidos (ACAD) propone, sin embargo, un diseño más flexible en cuanto es un modelo de enseñanza/aprendizaje basado en categorías de aprendizaje, es decir, basado principalmente en la adquisición de habilidades y el cambio de modelos mentales más que en la transmisión de información. El modelo ACAD refuerza los procesos de aprendizaje lingüístico desde el trabajo colaborativo y la importancia de la negociación en equipos desde valores de cooperación y respeto. Ello consiente reforzar uno de los principios del modelo de CCIC: integrar procesos de construcción social desde el aprendizaje de la lengua. La experimentación metodológica del modelo ACAD se ha planteado en entornos digitales para niveles de dominio B2C1con el propósito de integrar procesos de adquisición pragmática-discursiva en ELE vinculados a un proyecto lingüístico de sistematización del registro coloquial y la cortesía a través de actos de habla de petición (Leal Rivas, 2020a). El modelo instruccional ACAD ha flexibilizado los propósitos de la experimentación lingüística centrada, además, en mejorar la escritura académica formal asíncrona. El análisis del corpus textual de los participantes es actualmente en fase de análisis centrado en la adquisición de estrategias pragmáticas de cortesía valorizadora y atenuadora de los actos de petición en interacciones asíncronas (correo electrónico) y en relaciones de asimetría académica (profesor/alumno)

\section{Discusión resultados de implementación aplicativa de modelos DI para la CCIC en ELE}

La versatilidad de los modelos instruccionales responde de forma satisfactoria a la hora de estructurar a nivel aplicativo el modelo de CCIC y la interrelación de sus tres dimensiones (linguística, cognitiva y social) dentro de un enfoque competencial y de aprendizaje funcional y discursivo del español. El Diseño Instruccional por su naturaleza procesual favorece el desarrollo de habilidades cognitivas más complejas con prácticas discursivas más eficaces.

Los modelos de experimentación e investigación lingüística para el ámbito superior alojados en entornos virtuales proporcionan además un repositorio de corpus textuales (orales y escritos) que consienten a posteriori su análisis lingüístico y pragmático. Ello permite observar el preciso alcance y desarrollo de adquisición pragmática en ELE. 
Asimismo, los modelos instruccionales conforman un enfoque cognoscitivo asignado desde las estrategias que el alumno utiliza para procesar la información lingüística y discursiva, y que inciden en los procesos de comprensión crítica y de planificación, textualización y revisión de los textos. También reflejan los varios procesos que las redes digitales ofrecen en cuanto a "codificación, almacenamiento y recuperación de la información.” (Rovira Collado, 2016) que realizan los aprendientes, reforzando así una mayor interacción de las competencias no sólo comunicativas, lingüísticas o discursivas.

La adquisición pragmática en entornos virtuales de ELE se fomenta, además, desde actividades que se proponen como metas compartidas para maximizar el aprendizaje lingüístico también de forma cooperativa con la creación de grupos de conversación virtual (Chat), video clases, grupos virtuales de trabajo, etc.

La experimentación metodológica de los modelos instruccionales analizados proporciona ulteriores consideraciones que serían interesantes considerar para futuras experimentaciones cuyo objetivo sea integrar la adquisición pragmática en contextos digitales para un aprendizaje lingüístico en línea significativo y efectivo:

- El diseño de estrategias cognitivas directas o indirectas, implicadas en los procesos de aprendizaje de la lengua, deben integrar estrategias de interacción que asegure dinámicas colaborativas en línea entre docente-alumno y alumno-alumno.

- Fomentar la integración de mecanismos para favorecer la reflexión, el pensamiento crítico y la comprensión tanto del proceso de aprendizaje lingüístico como del propio proceso de aprendizaje en línea.

- Personalizar y adecuar el proceso de aprendizaje en línea adaptándolo a las necesidades de aprendizaje lingüístico de aprendientes con necesidades específicas para una mayor accesibilidad del aprendizaje de la lengua.

- Ofrecer siempre recursos y herramientas que refuerzan el proceso activo de enseñanza/aprendizaje de la lengua y favorece la interrelación y construcción social del grupo de aprendientes.

- El diseño estratégico de los entornos virtuales puede involucrar más tiempo a los aprendientes en contextos de aprendizaje lingüístico o de inmersión en lengua.

\section{Conclusiones}

En un contexto de gran vulnerabilidad como el que ha supuesto la pandemia causada por la Covid19 la reflexión a modo de cierre se propone hacia la contribución que la investigación linguística puede realizar en el contexto de la innovación educativa y digital vinculada a mejorar la formación lingüística en los distintos ámbitos educativos. El contexto actual está activando procesos de reflexión profunda en todos los niveles educativos y, en particular, la universidad debe asumir cómo integrar de forma realística y sistematizada en sus titulaciones multicompetencias que mejoren los procesos de aprendizaje lingüístico hacia nuevas alfabetizaciones, necesidades formativas y profesionales del siglo XXI.

Al hilo de las premisas y de las reflexiones desarrolladas en el estudio, se destaca la necesidad del paradigma interdisciplinar y transversal de la investigación lingüística aplicada a la enseñanza de lenguas en el contexto actual de una formación académica cada vez más especializada, plurilingüe y altamente competitiva. La formación permanente del docente es cada vez más compleja y diversificada, y por lo tanto, ha de ser altamente cualificada para responder a los estándares formativos de calidad.

En cuanto a los objetivos y el alcance que se habían propuesto se destacan las siguientes conclusiones deducibles de la reflexión articulada del presente estudio.

Mejorar la adquisición pragmática discursiva en entornos virtuales de enseñanza/aprendizaje de L2/LE supone experimentar enfoques metodológicos híbridos y modelos de enseñanza/aprendizaje lingüístico que cubran las necesidades discursivas y competenciales que los aprendientes deben afrontar en cualquier ámbito académico y en distintos contextos interculturales. Para ello, es importante considerar procesos de alfabetización mediática e informacional e integrar una competencia digital eficaz. En este sentido, el rol de la universidad como eslabón fundamental hacia la construcción social y la digitalización 
responsable debe reflejarse en una formación lingüística contextualizada y especializada que atienda a las necesidades discursivas académicas de los universitarios.

El modelo de CCIC destaca la necesidad de otorgar un mayor peso a los procesos de concienciación lingüística, discursiva, funcional e intercultural en la enseñanza/aprendizaje de lenguas. Dar cuenta de la dimensión intersubjetiva de los textos, considerar un enfoque discursivo del aprendizaje lingüístico desde estrategias y procesos cognitivos que se activan a través de la CCrítica y la Competencia Metapragmática, posibilita ir más allá de un aprendizaje formal y funcional de la lengua. El enfoque competencial del modelo de CCIC posibilita un "aprender a significar" en la lengua que implica un "saber comprometerse" socialmente con el uso de la lengua. Ello posibilita una mejor construcción social y un aprendizaje de valores que cabe integrar en la formación lingüística y académica universitaria.

Los modelos instruccionales analizados (ADDIE; IAP; ACAD) flexibilizan itinerarios de aprendizaje lingüístico y demuestran una gran adaptación para integrar una sistematización pragmática y discursiva en contextos digitales de ELE. Estos modelos revelan a través del análisis de corpus textuales realizados en distintas investigaciones lingüísticas el desarrollo consciente de estrategias pragmáticodiscursivas que vehiculan un uso discursivo crítico de la lengua.

El aprendiente de ELE es consciente de los valores y actitudes que puede transmitir a través de su construcción discursiva e ideológica de los textos, contribuyendo no solo a mejorar sus procesos de adquisición pragmático-discursiva en función de la tipología textual o género que utiliza, sino a ser agente activo en un proceso de construcción social desde su propio aprendizaje de la lengua.

\section{Referencias}

Álvarez Ramos, E. (2017). La didáctica de la lengua en entornos virtuales de aprendizaje: el caso concreto de la enseñanza-aprendizaje del español como lengua extranjera y la plataforma Eleclips. Revista de Educación a Distancia, 55, Artíc. 6. http://dx.doi.org/10.6018/red/55/6

Arzamendi, J. (2005). Comunicación y adecuación pragmalingüística. Funiber.

Baena, V. (2010). Innovación docente e identificación de inhibidores del aprendizaje en el área de empresa. Una propuesta metodológica. Espiral-Cuadernos del Profesorado, 3(6), 3-14. http://dx.doi.org/10.25115/ecp.v3i6.906

Bisquerra, R., Pérez, J.C., \& García Navarro, E. (2015). La inteligencia emocional en educación. Síntesis.

Byram, (2009). Multicultural Societies, Pluricultural People and the Project of Intercultural Education. https://www.coe.int/en/web/platform-plurilingual-intercultural-language-education/interculturalaspects\#\{"28072155":[0]\}

Campos Fernández-Fígares, M. (2013) Nuevas formas de leer II. Diccionario de nuevas formas de lectura y escritura. Álabe: Revista de Investigación sobre Lectura y Escritura, 7 (enero - junio), 1-43. http://revistaalabe.com/index/alabe/article/viewFile/174/13

Campos Fernández-Fígares, M. \& Quiles Cabrera, M.C. (2019) Repensando la didáctica de la lengua y la literatura: paradigmas y nuevas líneas de investigación. Visor Libros.

Cassany, D. (2017). Investigaciones y propuestas sobre literacidad actual. http://www2.udec.cl/catedraunesco/05CASSANY.pdf

Celce-Murcia, M., Dornyei, Z., \& Thurrell, S. (1995). Communicative Competence: A Pedagogically Motivated Model with Content Specifications. Issues in Applied Linguistics, 6(2). https://escholarship.org/uc/item/2928w4zj

Consejo de Europa (2002). Marco Europeo Común de Referencia para las Lenguas: Aprendizaje, Enseñanza, Evaluación. [Traducido por el Instituto https://cvc.cervantes.es/ensenanza/biblioteca_ele/marco/indice.htm

Cuenca, M.J. \& Hilferty, J. (1999). Introducción a la lingüística cognitiva. Ariel.

De León C. I. y Suárez N. J. (2008). El Diseño Instruccional y Tecnologías de la Información y la Comunicación. Posibilidades y Limitaciones. Revista de Investigación, 65, 57-81. Universidad Pedagógica Experimental Libertador. 
Díaz Barriga, F. (2006). Principios de diseño instruccional de entornos de aprendizaje apoyados en TIC: un marco de referencia sociocultural y situado. Tecnología y Comunicación Educativa, 41. http://investigacion.ilce.edu.mx/tyce/41/art1.pdf

Elliott, J. (1993). El cambio educativo desde la investigación-acción. Morata.

Fontich, X. (2006). Hablar y escribir para aprender gramática. Cuadernos de educación, 50. Horsori. http://www.xtec.cat/ ilopez15/materials/gramatica/hablaryescribirparaaprendergramatica.pdf

Galindo Merino, M. del Mar (2016). La lengua materna en el aula de ELE. Biblioteca Virtual Miguel de Cervantes. Http://www.cervantesvirtual.com/nd/ark:/59851/bmcrn564

Halliday, M. (1982). El lenguaje como semiótica social. Fondo de Cultura Económica.

Halliday M. \& Hasan R. (1989). Language, Context and Text: Aspects of Language in a Social-Semiotic Perspective. Deakin University Press.

Halliday M. \& Matthiessen C. (2004). An Introduction to Functional Grammar. Arnold.

Instituto Cervantes (2006). Plan curricular del Instituto Cervantes. Niveles de referencia para el español. Instituto Cervantes - Biblioteca nueva. http://cvc.cervantes.es/ensenanza/biblioteca_ele/plan_curricular/

Leal-Rivas, N. (2018). Aprendizaje consciente en entornos digitales: estrategias cognitivas para una competencia comunicativa intercultural. En Chiapello, S. et al. (Eds.), Telecolaboración y corpus para el estudio de lengua y cultura, 169-184. Servei de Publicacions de la Universitat d'Alacant. https://dialnet.unirioja.es/servlet/libro?codigo=726709

Leal-Rivas, N. (2019). Construcción social y ELE: reflexión metalingüística, interculturalidad y competencia pragmático-discursiva en estudiantes de movilidad. Pedagogía Socia. Revista Interuniversitaria, 33, 31-43. https://doi.org/10.7179/PSRI_2019.33.05

Leal-Rivas, N. (2020a). La competencia lecto-crítica en entornos digitales: una visión holística para L2/LE. Contextos Educativos, 25, 71-89. http://doi.org/10.18172/con.4267

Leal-Rivas, N. (2020b). Competencia Comunicativa Intercultural Crítica y escritura académica: análisis de los (sub)procesos de creación textual en estudiantes universitarios de Español Lengua Extranjera (E/LE). Porta Linguarum. An International and Interuniversity Journal of Foreign Language Teaching and Learning, 34, 169-192. https://doi.org/10.30827/portalin.v0i34.16739

López Gómez, E. \& Pérez Navío, E. (2013). Formación permanente del profesorado y práctica docente intercultural: contenidos actitudinales y complementariedad competencial. Espiral-Cuadernos del Profesorado, 6 (12), 32-42. http://dx.doi.org/10.25115/ecp.v6i12.955

Marco Europeo de Competencias Digitales para la Ciudadanía-DigComp 2.1 https://publications.jrc.ec.europa.eu/repository/bitstream/JRC106281/web-digcomp2.1pdf_(online).pdf

Marco Europeo de Competencias Digitales para Educadores-DigCompEdu https://ec.europa.eu/jrc/sites/jrcsh/files/digcompedu_leaflet_en-2017-11-14.pdf

Marimón Llorca, C. (2016). Hacia una dimensión crítica en la enseñanza de español como lengua extranjera: La Competencia Comunicativa Intercultural Crítica (CCIC). Revista española de lingüística aplicada, Vol. 29, Vol. 1, 2016, 191-21. https://dialnet.unirioja.es/ejemplar/456371

Martí, J. (2002). La investigación-acción-participativa. Estructuras y fases. En Rdz. Villasante et al. La investigación social participativa. Construyendo ciudadanía 1: El viejo topo, 123).http://www.redcimas.org/wordpress/wp-content/uploads/2012/08/m_JMarti_IAPFASES.pdf

Martínez Ezquerro, A. (2020). Habilidades lingüísticas en prácticas lectoras interculturales. Porta Linguarum. Revista Internacional de Didáctica de las Lenguas Extranjeras, 34 (junio 2020), 107-123. https://doi.org/10.30827/portalin.v0i34.16736

Martínez Ezquerro, A. (2020). Aplicaciones lingüístico-comunicativas para la interculturalidad. En Del multiculturalismo a los mundos distópicos. Temas actuales de la didáctica de la lengua y la literatura. $\left(1^{\mathrm{a}}\right.$ ed., 29-44). Marcial Pons, Colección "Universidad y Lectura.

Martos Núñez, E. y Campos, M. (Coords.) (2013). Diccionario de nuevas formas de lectura y escritura. Santillana.

Níkleva, D. G. y Rodríguez Muñoz, F. (2015). Renovación metodológica en el Espacio Europeo de Educación Superior. Espiral. Cuadernos del Profesorado, 8 (17), 3-15. http://dx.doi.org/10.25115/ecp.v8i17

Pastor Cesteros, S. (2004). El papel de la reflexión metalingüística en la adquisición de la gramática de E/LE. En $X V$ Congreso Internacional de ASELE. Las gramáticas y los diccionarios en la enseñanza del español como segunda lengua, deseo y realidad, Universidad de Sevilla. https://idus.us.es/xmlui/handle/11441/42547 
Portolés, J. (2004). Pragmática para hispanistas. Síntesis.

Quiles Cabrera, M.C. \& Caire, M-P. (2013) Sobre el texto y la competencia cultural en la Educación Superior: aproximación a un aula de ELE. Porta Linguarum: revista internacional de didáctica de las lenguas extranjeras, 19, 2013, 199-218.

Quiles Cabrera, M.C. \& Campos Fernández-Fígares, M. (2015) Una aproximación empírica al español escrito por escolares inmigrados. Revista Internacional de Estudios Migratorios (RIEM), Vol. 5, №. 2, 172-200.

Recomendación del Consejo, de 22 de mayo de 2018, relativa a las competencias clave para el aprendizaje permanente https://eur-lex.europa.eu/legal-content/ES/TXT/?uri=CELEX:32018H0604(01)

Robles Ávila, S. (2019). A vueltas con el enfoque orientado a la acción: leyendo las nuevas aportaciones desde el volumen complementario del MCER (2017). Álabe 19, 1-17. http://dx.doi.org/10.15645/Alabe2019.19.10

Rodríguez Muñoz, F.J. (2010). La competencia en comunicación lingüística a partir de la corrección de textos periodísticos: una experiencia docente. Espiral. Cuadernos del Profesorado, 3(6), 53-64. Doi: http://dx.doi.org/10.25115/ecp.v3i6.910

Rovira Collado, J. (2016). Redes sociales en la universidad: profesionales, académicas y de lectura. Álabe 13, (enerojunio). Doi: 10.15645/Alabe2016.13.4

Rutherford, W. (1987). Second Language Grammar: Learning and Teaching. Longman.

Van Dijk, T.A. (1999). El análisis crítico del discurso. Anthropos, 186. 23-36. http://www.discursos.org/oldarticles/El\%20an\%E1lisis\%20cr\% EDtico\%20del \%20discurso.pdf

Van Dijk, T. Discurso y manipulación: Discusión teórica y algunas aplicaciones. Revista Signos, 2006, vol.39, n.60, 49-74. http://dx.doi.org/10.4067/S0718-09342006000100003 